\title{
Five year refractive outcome of LASIK for myopia and myopic astigmatism in Vietnam
}

\author{
Thanh Pham ${ }^{1,2}$, Con Petsoglou ${ }^{2,3}$, Yen Tran Hai ${ }^{1,4}$ \\ ${ }^{1}$ Haiyen Eye Center, Ho Chi Minh City, Vietnam; ${ }^{2}$ Save Sight Institute \& Discipline of \\ Clinical Ophthalmology, University of Sydney, Sydney, New South Wales, Australia; \\ ${ }^{3}$ Lions NSW Eye Bank, Sydney, New South Wales, Australia; ${ }^{4}$ Ho Chi Minh City Eye \\ Hospital, Ho Chi Minh City, Vietnam.
}

\begin{abstract}
Purpose: To assess the long-term refractive outcome of laser in situ keratomileusis (LASIK) for myopia and myopic astigmatism

Design: An observational, single center, multisurgeon case series

Methods: This study was a follow up study of 128 patients contacted to return for repeat investigation post refractive surgery. It included 245 eyes of 128 patients who underwent LASIK for myopia or myopic astigmatism at the Ho Chi Minh City Eye Hospital, Vietnam in 2008. Surgical parameters as well as patient data at presentation, three months and five years after surgery were analyzed. The main outcome was spherical equivalent (SE) within $\pm 0.5 D$ at 5 years.

Results: At the fifth year follow up, $80 \%$ of eyes were within $\pm 0.5 \mathrm{D}$ and $98 \%$ of eyes were within $\pm 1.0 \mathrm{D}$ of emmetropia. No eyes underwent laser enhancement prior to the last visit although the retreatment criteria was met in 45 eyes (18.4\%). The mean SE decreased from $0.16 \pm 0.33$ at 3 months to $-0.07 \pm 0.50$ at 5 years for the whole cohort. Patients with severe myopia were most susceptible to myopic regression and had a less predictable refractive outcome. The overall UCVA of 20/20 or better and of 20/40 or better were obtained in $68.2 \%$ and $95.1 \%$ of eyes. Fourteen eyes (5.7\%) improved 1 line of BCVA. Forty two eyes (17.1\%) lost 2 lines of BCVA which were unrelated to laser complications.

Conclusion: Although there was myopic regression over time, LASIK provided excellent 5 year refractive outcome for myopia and myopic astigmatism. Almost $20 \%$ of patients meet criteria for retreatment but do not seek a retreatment in this Vietnamese population.
\end{abstract}

Keywords: LASIK, refractive outcome, myopia, myopic astigmatism

\section{Introduction}

Myopia is the most common eye condition worldwide, which affects up to $80 \%$ of people in some Asian populations. ${ }^{1,2}$ For most patients, glasses and contact lenses are the most convenient way of correcting myopia. The most commonly performed operative procedure for myopia is LASIK (Laser In situ Keratomileusis). This is a relatively safe procedure with quick visual recovery. More than 28 million LASIK surgeries have been performed globally with a patient satisfaction rate regarding refractive efficacy of over $95 \% .^{3,4}$

Since the foremost aim of LASIK surgery is to correct refractive error in order to reduce dependence on eye glasses or contact lenses, its long term refractive

Correspondence: Thanh Ngoc Dan Pham, Haiyen Eye Center, 31A Nguyen Dinh Chieu street, Dakao ward, district 1, Ho Chi Minh City, Vietnam.

E-mail: thanhpham1908@gmail.com 
outcome is of great concern. ${ }^{3}$ Most published reports of refractive efficacy are limited to 1 year with only a few reports beyond 5 years. ${ }^{5-12}$ These studies in general report that postoperative refraction does remain stable beyond 5 years.?

In Vietnam, LASIK was first introduced in 2000. The long term follow up for postoperative outcomes has not been well studied in our setting. Locally a final postoperative visit occurs at 3 months with patients then they are lost to follow up. Thus, the aim of this study was to assess the long term (5 year) refractive outcome of uneventful LASIK for myopia and myopic astigmatism in Vietnamese patients. These results were compared to the 3 month results at all levels of myopia. As a secondary aim, the study also looked into the association between the 5 year refraction and predictors of regression.

\section{Materials and methods}

\section{Patient population}

This study involved contacting and recruiting patients who underwent LASIK surgery at the Ho Chi Minh City Eye Hospital (HCMCEH) in 2008.

The criteria for LASIK eligibility at the HCMCEH since 2008 were: patients aged 18 or older with manifest spherical equivalent (SE) refraction less than or equal to -12.0 diopters $(D)$, astigmatism less than or equal to $-6.0 \mathrm{D}$, difference between best corrected visual acuity (BCVA) and uncorrected visual acuity (UCVA) equal or more than 2 lines, and stable refraction for at least 6 months before surgery. The exclusion criteria for surgery were: any past or present ocular diseases or systemic diseases that might lead to abnormal wound healing, insufficient corneal thickness for laser ablation (total corneal thickness $<475 \mu \mathrm{m}$ ), evidence of keratoconus or keratoconus suspect, untreated retinal breaks, and women who were pregnant or 12 months postpartum.

Inclusion criteria for our study were: 1. Resident in Ho Chi Minh City, 2. Underwent uneventful LASIK for myopia and myopic astigmatism, 3. Complete postoperative information up to 3 months. Of the 3922 patients who underwent LASIK in 2008, 772 met these criteria. Reasons for study exclusion of 3150 patients were due to them living outside Ho Chi Minh City, having other refractive errors or incomplete 3 month follow up data. Patients were contacted by letters inviting them to participate and/or phone messages. Of the 772 individuals, 148 patients (19.2\%) agreed to participate and scheduled a free follow up assessment. Twenty patients were excluded from the analysis for not completing all the required tests (3 patients) or having unreliable data in any studied parameters (17 patients) at the 5 year visit. Our study then was left with 245 eyes of 128 patients which were analyzed.

Informed consent was obtained from all patients for their initial surgery and this follow up study. 


\section{Patient management}

\section{Preoperative Examination}

Prior to the surgery, all patients underwent detailed examination included UCVA, BCVA (Auto Chart Projector, Shin Nippon), manifest and cycloplegic refraction (Welch Allyn retinoscope and Inami refraction lens case), contrast sensitivity (FACT, Stereo Optical), corneal topography (Orbscan, Technolas Perfect Vision), corneal pachymetry (AL2000, Tomey), preoperative intraocular pressure testing (IOP) (Schiotz, Germany), and slitlamp biomicroscopy examination (Topcon, Japan).

\section{Surgical Procedure}

LASIK procedures were performed by surgeons of the HCMCEH, using the same technique and protocol. Eyelids were retracted with a speculum. After the eye was positioned beneath the laser, alignment marks were placed on the cornea. Superior-hinged flap was created by $\mathrm{M} 2$ automated microkeratome (Moria) or Hansatome (Bausch and Lomb), based on nomograms provided by the manufacturer. The flap was reflected and excimer laser ablation was then performed with Ladarvision, WaveLight Allegretto (Alcon), or Technolas 217z (Technolas Perfect Vision) laser systems. After ablation, the interface was irrigated with balanced salt solution and then replaced. The striae test was performed to check flap adhesion before removing speculum.

\section{Postoperative Evaluation}

Following surgery, topical antibiotic (Ofloxacin $0.3 \%$, Santen) was used for the first week. Corticosteroid eye drops (Pred Forte 1\%, Allergan) was prescribed for 1 week. Nonpreservative artificial tear was used for up to 3 months.

Patients underwent postoperative examinations at 1 day, 1 week, 1 month, 3 months and 5 years (this study) after the initial procedure by independent examiners. Slit lamp examination and UCVA were performed at all examinations. Manifest refraction, BCVA and contrast sensitivity were assessed from the 1 week recheck. At 5 years, IOP and corneal thickness were remeasured by using ocular response analyzer (Reichert) and pachymeter (AL2000, Tomey). Flap thickness measurement was done with the optical coherence tomography (OCT1000, Carl Zeiss). Chorioretinal degenerative signs were recorded. Residual stromal bed thickness was calculated by using preoperative pachymetry minus predicted flap thickness minus calculated ablation depth.

\section{Variable measurements}

\section{Outcome}

Spherical equivalent of BCVA at 5 years was the primary outcome of interest. It was examined as a continuous variable and then grouped into 2 groups based on its value within $\pm 0.5 \mathrm{D}$ range. 


\section{Exposed (predictor) variables of interest:}

Levels of myopia (based on manifest SE) were grouped into 3 levels: mild ( 0 to $-3.0 \mathrm{D})$, moderate $(-3.12$ to $-6.0 \mathrm{D})$, and severe (more than $-6.0 \mathrm{D})$. Regression was defined as a $0.5 \mathrm{D}$ or more myopic shift occurring between follow up visits without laser enhancement. The 3 month regression was further categorized into whether or not regressed at 3 months to predict for the 5 year refractive outcome. Operative machine (Ladarvision, Allegretto and Technolas) was also included. Safety index $=\mathrm{BCVA}_{\text {postoperative }} / \mathrm{BCVA} \mathrm{preoperative}$ Efficacy index $=\mathrm{UCVA}_{\text {postoperative }} / \mathrm{BCVA}$ preoperative . Corneal haze was graded from 0 to 4 in which grade 0 (clear cornea) and 1 (trace haze) were then grouped as insignificant, and the remaining grades were significant haze group. Degree of lens opacity was graded according to the LOCS III system after pupil dilatation. A gradable cataract was defined as grade 3 or more in nuclear or cortical cataract, or a grade 2 or more posterior subcapsular cataract ${ }^{13}$. Age at surgery, preoperative pachymetry value, flap thickness, optic zone, ablation depth and residual stromal bed thickness were all examined by regression analysis for any association with postoperative SE at 5 years.

\section{Other variable description:}

Undercorrection was defined as an SE of $-0.5 \mathrm{D}$ or more at the first reassessment of postoperative refraction (at 1 week visit). Retreatment criteria, after the first 3 months, included any of the following parameters: manifest SE of $-0.5 \mathrm{D}$ or more, UCVA of $20 / 32$ or less, stable myopic refraction between consecutive visits, and patient dissatisfaction with the visual result including sufficient residual stromal bed thickness. Snellen acuity, noted in decimal, was converted to the logarithm of the minimum angle of resolution (LogMAR) equivalent for analysis and back to lines for interpretation. Changes in visual acuity and refraction at 3 months and 5 years were compared between three levels.

\section{Statistical analysis}

All variables were assessed through univariate analysis. Bivariate analysis was conducted to compare differences in some measurements according to level of myopia ( $t$ test or chi square test). In order to predict $S E$ at 5 year range within $\pm 0.5 \mathrm{D}$, multivariate analysis was used to assess the role of the given exposure variables in predicting the refractive outcome. Logistic regression was used to select the best subset of predictors. Data was entered using Excel 10 and analyzed using SAS 9.4 at 0.05 level of significance.

\section{Results}

\section{Demographics of patients and surgical related parameters}

In this study, 245 eyes of 128 patients that underwent LASIK surgery for myopia and myopic astigmatism were analyzed. There was no age difference of the patients within mild, moderate and severe myopia group $(p=0.33)$. Demographics of the patients are listed (Table 1). 
Table 1 Demographics of patients included in this study

\begin{tabular}{|l|l|}
\hline Gender (\%) & \\
\hline Male & $63(25.7)$ \\
\hline Female & $182(74.3)$ \\
\hline Myopia levels (\%) & \\
\hline Mild ( $\leq 3.0 D)$ & $51(20.8)$ \\
\hline Moderate (-3.12 to -6.0D) & $128(52.2)$ \\
\hline Severe (>6.0D) & $66(27)$ \\
\hline Mean age \pm SD, range, years & $25.0 \pm 6.3(18.0$ to 43.0$)$ \\
\hline Mean SE \pm SD, range, D & $-4.99 \pm 2.3(-12.0$ to -0.75$)$ \\
\hline Mean corneal thickness \pm SD, range, $\mu m$ & $524.97 \pm 31.1(475$ to 609$)$ \\
\hline SD = standard deviation; SE $=$ spherical equivalent; $D=$ diopters \\
\hline
\end{tabular}

All eyes were targeted for emmetropic correction. Technolas $217 z$ was used in $193(78.8 \%)$ operations while Ladarvision and Allegretto were used in 29 (11.8\%) and $23(9.4 \%)$ cases, respectively. Diameter of the ablated area was $6.5 \pm 0.1 \mathrm{~mm}$ in the optical zone, and $6.6 \pm 0.4 \mathrm{~mm}$ when the transitional zone was included. The mean ablation depth was $91.7 \pm 29.3 \mu \mathrm{m}$. Laser ablation left an average residual stromal bed thickness of $310 \pm 36.5 \mu \mathrm{m}$. Postoperative corneal thickness was 463.8 $\pm 39.9 \mu \mathrm{m}$. At 5 years, gradable cataracts were present in 7 cases (2.9\%). Clear corneas were found in $88.2 \%$ of patients postoperatively, while haze graded 1 and grade 2 were only in 26 cases $(10.6 \%)$ and 3 cases $(1.2 \%)$, respectively. IOP at the $5^{\text {th }}$ year visit was $14.1 \pm 2.7 \mathrm{mmHg}$.

\section{Refractive outcome, predictability and stability}

The manifest refraction of $-4.99 \pm 2.27 \mathrm{D}$ preoperatively was improved to $0.16 \pm$ $0.33 \mathrm{D}$ at 3 months. The manifest refraction at 5 years was slightly reduced to -0.07 $\pm 0.50 \mathrm{D}$ compared to the 3 month average. Refractive results declined over time in all three levels of myopia (Table 2). 
Table 2 Spherical equivalent (SE) refraction over 5 years stratified by levels of myopia

\begin{tabular}{|l|c|c|c|c|}
\hline & No of eyes & Preop SE* & SE at 3 months & SE at 5 years* \\
\hline Mild (<3.0D) & 51 & $-2.19 \pm 0.59$ & $0.18 \pm 0.28$ & $0.06 \pm 0.45$ \\
\hline Range, D & & -0.75 to -3.0 & -0.25 to +1.00 & -1.00 to +1.00 \\
\hline Median & & -2.25 & 0.25 & 0.00 \\
\hline $\begin{array}{l}\text { Moderate } \\
\text { (-3.12 to -6.0D) }\end{array}$ & 128 & $-4.58 \pm 0.79$ & $0.19 \pm 0.26$ & $0.01 \pm 0.42$ \\
\hline Range, D & & -3.12 to -6.0 & -0.38 to +1.00 & -1.25 to +0.75 \\
\hline Median & & -4.56 & 0.25 & 0.00 \\
\hline Severe (>-6.0D) & 66 & $-7.94 \pm 1.60$ & $-0.25 \pm 0.46$ & $-0.32 \pm 0.60$ \\
\hline Range, D & & -6.13 to -12.0 & -1.38 to +1.75 & -3.13 to +0.75 \\
\hline Median & & -7.44 & 0.00 & -0.25 \\
\hline All levels & 245 & $-4.99 \pm 2.27$ & $0.16 \pm 0.33$ & $-0.07 \pm 0.50$ \\
\hline Range, D & & -12.00 to -0.75 & -1.38 to +1.75 & -3.13 to +1.00 \\
\hline $\begin{array}{l}\text { SE = spherical equivalent; SD }=\text { standard deviation; D }=\text { diopters } \\
\text { *Test differences between means of myopia level: } p<0.0001\end{array}$ & \\
\hline
\end{tabular}

The overall percentage of myopic regression increased from $16 \%$ at 3 months to $30 \%$ at the 5 year visit. Eyes in the severe myopia group had the highest percentages of myopic regression over time and means of SE at 3 months and at 5 years were significantly different by level of myopia (Fig.1).

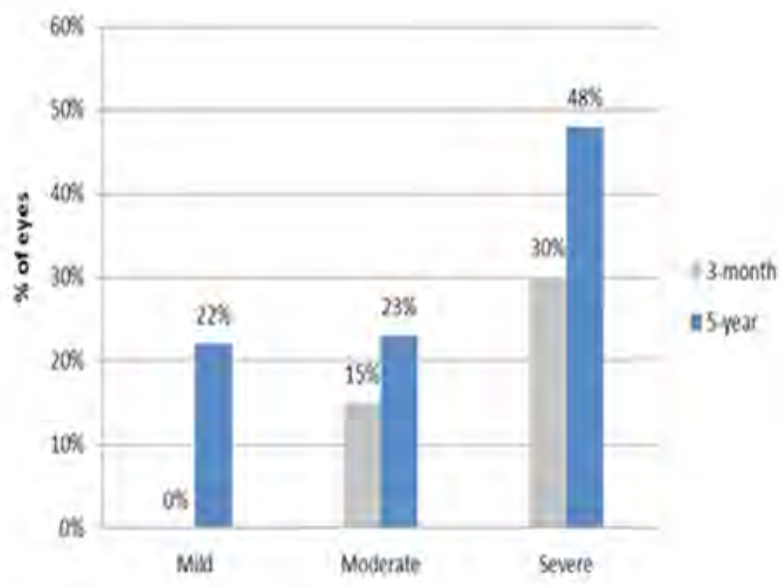

Fig 1. Myopic regression in different levels of myopia 
At 5 years, the mean regression in each level of myopia were $-0.12 D$ (mild), $-0.18 D$ (moderate) and $-0.4 \mathrm{D}$ (severe). Regarding to the refractive predictability, the overall prevalence of eyes achieving SE within $\pm 0.5 \mathrm{D}$ declined by more than 10 percentage point after 5 years (from $93 \%$ to $80 \%$ ), whereas prevalence of SE within $\pm 1.0 \mathrm{D}$ remained almost the same at $99 \%$ during the period (Table 3 ).

Among three types of operative machine, the rate of regression at 5 years after Ladarvision (62\%) was two times higher than Technolas (24\%) and Allegretto (30\%).

Table 3 Prevalence of eyes within $\pm 0.5 \mathrm{D}$ or $\pm 1.0 \mathrm{D}$ of target correction stratified by levels of myopia

\begin{tabular}{|r|l|l|l|l|}
\hline & Within \pm 0.5 D & Within \pm 1.0 D & & \\
\hline & At 3 months & At 5 years & At 3 months & At 5 years \\
\hline Mild & $96 \%$ & $80 \%$ & $100 \%$ & $100 \%$ \\
\hline Moderate & $95 \%$ & $84 \%$ & $100 \%$ & $99 \%$ \\
\hline Severe & $89 \%$ & $70 \%$ & $95 \%$ & $94 \%$ \\
\hline All levels & $93 \%$ & $80 \%$ & $99 \%$ & $98 \%$ \\
\hline
\end{tabular}

Factors associated with the 5 year SE within $\pm 0.5 \mathrm{D}$

Demographics of the eyes achieving SE within $\pm 0.5 \mathrm{D}$ at 5 years following LASIK had a mean age of $24.9 \pm 6.3$, mean SE of $-5.01 \pm 2.27$. The myopia level distribution was $19 \%$ (mild), $55.4 \%$ (moderate) and $25.6 \%$ (severe). Technolas $217 z$, Ladarvision and Allegretto were used in $81.5 \%, 8.2 \%$ and $10.3 \%$ of cases, respectively.

Among various variables, age at surgery was the only factor having association with 5 year SE within $\pm 0.5 \mathrm{D}$ after adjustment (Table 4). Specifically the younger patients tended to regress more than older patients. 
Table 4 Associations between the 5 year SE predictability within $\pm 0.5 \mathrm{D}$ and exposed variables

\begin{tabular}{|r|l|}
\hline & Adjusted OR $(95 \% \mathrm{Cl})$ \\
\hline Age at surgery & $0.93(0.88,0.98)$ \\
\hline Myopia level & $1.17(0.43,3.17)$ \\
\hline Preoperative corneal thickness & $0.99(0.96,1.03)$ \\
\hline Flap thickness & $0.96(0.91,1.01)$ \\
\hline Optic zone & $4.04(0.32,51.28)$ \\
\hline Ablation depth & $0.99(0.95,1.03)$ \\
\hline Machine type & $1.14(0.64,2.02)$ \\
\hline Regression at 3 month & $1.14(0.43,3.02)$ \\
\hline Residual stromal bed thickness & $1.00(0.98,1.04)$ \\
\hline Cataract & $2.07(0.26,16.37)$ \\
\hline Haze & $(0.22,1.10)$ \\
\hline &
\end{tabular}

Visual outcome, efficacy and safety

At the fifth year post-operation, the efficacy index was 0.96 and the safety index was 1.07. Both were lower than its parameters at 3 months which were 1.24 and 1.29. The preoperative UCVA was $1.12 \pm 0.21$ (range, 0.40 to 1.70 ), improved to -0.07 at 3 months, and reached 0.03 at 5 years after surgery. The overall final UCVA of $20 / 20$ or better and of $20 / 40$ or better were obtained in $68.2 \%$ and $95.1 \%$ of patients, respectively. About $50 \%$ of the eyes with severe myopia retained UCVA of $20 / 20$ or better at the last checkup (Fig. 2).

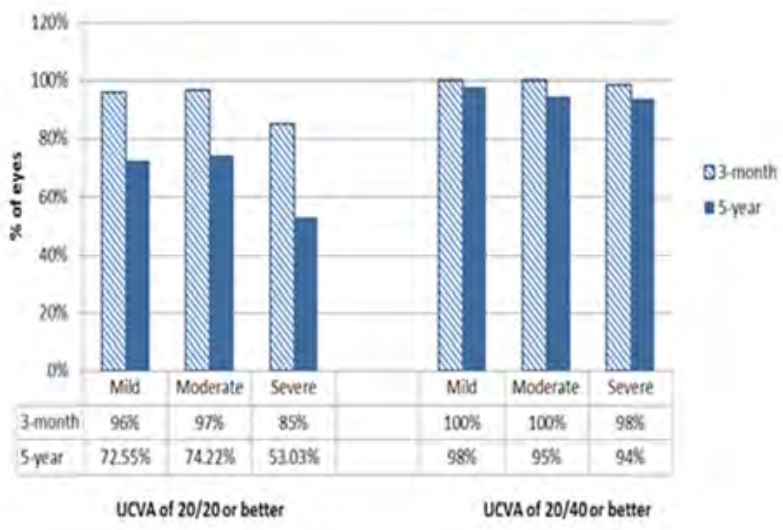

Fig 2. UCVA outcomes at 3 months and 5 years postoperation in different levels of myopia 
The 5 year BCVA was unchanged in 82 eyes (33.5\%), improved 1 line in 14 eyes (5.7\%) and dropped 1 to 2 lines in 149 eyes (60.8\%). In the 42 eyes (17.1\%) that lost 2 lines of BCVA, the reasons were due to progressive cataract (7\%) and myopic chorioretinal degeneration (93\%).

No eye underwent laser enhancement prior to the last visit although the indication for retreatment was met in 45 eyes (18.4\%) at the fifth year.

There were 4 cases that the patient underwent LASIK on one eye only. The other eyes progression of myopia was almost the same with the operated one (difference of $0.25 \mathrm{D}$ )

\section{Discussion}

In this study, we showed a mild myopic regression at the 5 year refractive outcome of uneventful LASIK for myopia and myopic astigmatism at HCMCEH.

Refractive outcome, predictability and stability

After surgery, the mean SE changed, from $0.16 \pm 0.33 \mathrm{D}$ at 3 months to $-0.07 \pm$ $0.50 \mathrm{D}$ at 5 years, indicating a myopic shift over time. The overall percentage of eyes having myopic regression doubled over the period. This was seen more in the severe group with two to three times more than that of the other two groups. This tendency has been confirmed in a number of other prospective case series studying the mechanism of post LASIK regression. ${ }^{14}$ However, Liu Z LY, in a seven year study with moderate to severe myopia found that eyes did not regress in refraction between 1 and 7 years postoperatively. ${ }^{9}$

Regarding to the predictability of refractive surgery, we found that $80 \%$ of eyes retained within $\pm 0.5 \mathrm{D}$ of target correction and $98 \%$ were within $\pm 1.0 \mathrm{D}$ at the fifth year. Our $0.5 \mathrm{D}$ refractive predictability result was similar to those of previous researches which range from $60 \%$ to $89 \% .^{9-11}$ In terms of the $1.0 \mathrm{D}$ refractive predictability, although several studies showed comparable figures to $u s_{,}^{6,7,9-11}$ some six to eleven year follow up reports revealed a predictability of around $50 \% .5,12,15$ When looking at each group, severe myopic eyes had a less predictable outcome (70\%) than eyes with mild to moderate myopia (from $84 \%$ ). These results were comparable with findings in a 13 year follow up study. ${ }^{11}$

Factors associated with the 5 year SE within $\pm 0.5 \mathrm{D}$

In previous studies, myopic regression mechanisms were reported due to nuclear sclerosis of the lens, corneal steepening due to the thinning, corneal hydration, stromal synthesis, and compensatory epithelial hyperplasia. ${ }^{14}$ In addition, higher achieved correction and change in corneal power were also found to be associated factors of regression. ${ }^{7}$ This study, after adjusting for a number of factors, we observed a significant association between age at surgery and the 5 year SE within $\pm 0.5 \mathrm{D}$ of target correction. Our result implied that with each year increase in age, patients face a $5 \%$ possibility of having their 5 year SE outside the 0.5 range, OR = $0.93(0.88,0.98)$. 


\section{Visual outcome, efficacy and safety}

In the long term, our results indicated that both efficacy and safety index of uneventful LASIK followed a downtrend. However, its safety parameter remained higher than 1.0. This outcome was reinforced by the same results of the 10 years $^{7}$ and 15 years follow up studies (M.Elbahrawy et al., the XXXII Congress of ESCRS 2014).

At the fifth year, almost all eyes (95\%) could be independent of spectacles with the UCVA of 20/40 or better. Our findings were close to the results of some studies (between $78 \%$ to $100 \%),{ }^{6,79-11}$ but it more than double that in three reports (33\% to $46 \%$ ) which conducted on moderate to extreme myopia ${ }^{5,12}$ (M. Elbahrawy et al., the XXXII Congress of ESCRS 2014). In our study, about three fourths of eyes in the mild and moderate group retained their UCVA of 20/20 at 5 years whereas this amount in the severe group was just over a half. When we compare these results with the figures at 3 months, a $20-30 \%$ decrease was noted in each level of myopia regarding to the probability of obtaining UCVA of 20/20 or better over time.

BCVA was unchanged in $33.5 \%$ of eyes, and about $5.7 \%$ of eyes gained 1 line at 5 years. According to Applegate, the improvement in BCVA may be due to the change in the plane of correction, from the spectacle to the cornea, which induced a retinal image magnification effect. Other long term studies also reported positive final BCVA of their patients. ${ }^{5-7,9,10,12}$ Among three levels of myopia in our study, the moderate group achieved the highest rate in BCVA improvement.

To the best of our knowledge, this is the first study in Vietnam to evaluate the 5 year refractive outcome in LASIK patients in terms of regression and SE range within $0.5 \mathrm{D}$ of target correction. We also attempted to look at predictors of myopic regression across a number of clinical variables. To us, the 3 month landmark is very important as it is not only the least time for postoperative refraction stabilization ${ }^{16}$ but also the last time most of our patients returned for reassessment. The outcomes were described in separate levels of myopia, which is clinical relevant in postoperative LASIK studies.

However, this study had several limitations. This was a follow-up study by invitation. The 5-year follow up group was only 128 of 3922 patients who had LASIK at our center (3.3\%). There was a large loss to follow up due to patients living outside Ho Chi Minh City and were unable to be reexamined. Of the 772 patients eligible to be recruited, only 128 agreed (17\%). This small study group does result in recruitment bias for our results. Further, our study involves treatment by a number of different LASIK machines which may have themselves have different long term refractive stability outcomes. Despite this, the type of operative machine (Allegretto, Technolas and Ladarvision) showed no significant difference in postoperative SE nor safety and efficacy ${ }^{17,18}$.

In conclusion, our findings showed that although there was a regression in postoperative results over time, uneventful LASIK for myopia and myopic astigmatism provided a good long term refractive outcome at 5 years after surgery. About $95 \%$ of eyes could be independent of their glasses (UCVA of 20/40 or better) and nearly $70 \%$ retained emmetropia (UCVA of $20 / 20$ or better). $17 \%$ of eyes lost 2 lines 
of BCVA due to progressive cataracts and myopic retinal degeneration. Approximately $80 \%$ and $98 \%$ of eyes were within $\pm 0.5 \mathrm{D}$ and $\pm 1.0 \mathrm{D}$ at 5 years. Severe myopia was most susceptible to myopic regression and had less predictable outcome as compared to mild and moderate myopia.

\section{Acknowledgements}

Thank the Board of Directors and the Refractive Surgery Department of the Ho Chi Minh City Eye Hospital, Vietnam for their facilitation.

Thank Dr. Pham Trong Van, the vice head of Ophthalmology Department of Hanoi Medical University, for his encouragement and comments.

Acknowledge the support in data collecting activities from Mr. Trung Nguyen Thien and Mr. Tri Trinh Quang.

Appreciate Mrs. Trang ND Pham for her statistical expertise.

\section{References}

1. Lee YY LC, Sheu SJ, Lin JL. Factors Associated with Myopia in Young Adults. IOVS. 2013;54(2):1026-1033.

2. Pan C-W RD, Saw S-M. Worldwide prevalence and risk factors for myopia. Ophthalmic \& Physiological Optics. 2012(32):3-16.

3. Solomon K.D LEFdC, Helga P. Sandoval, Joseph M. Biber, Brian Groat, Kristiana D. Neff, Michelle S.Ying, John W.French, Eric D.Donnenfeld, Richard L.Lindstrom. LASIK World Literature Review. Ophthalmology. 2009;116(4):691-701.

4. Stuart A. A look at Lasik Past, Present and Future. Eyenet: American Academy of Ophthalmology; 2009:39-44.

5. George D. KymionisI NST, Nikolaos Astyrakasis, et al. Eleven-year follow up of laser in situ keratomileusus. J Cataract Refract Surg. 2007;33:191-196.

6. Ide T et al. Outcome of a 10-year follow-up of laser in situ laser keratomileusis for myopia and myopic astigmatism. Taiwan Journal of Ophthalmology. 2014:1-7.

7. Jorge L. Alio OM, Dolores Ortiz, et al. Ten Year Follow-up of Laser in Situ Keratomileusis for Myopia of up to -10 Diopters. American Journal of Ophthalmology. 2008;145(1):46-54.

8. Kato N TI, Hori-Komai Y, Sakai C, Tsubota K. Five-Year Outcome of LASIK for Myopia. Ophthalmology. 2008;115(5):839-844.

9. Liu Z LY, Cheng Z, et al. Seven-year follow up of LASIK for moderate to severe myopia. J Refract Surg. 2008;24(9):935-940.

10. M O'Doherty MOK, C Kelleher. Five year follow up of laser in situ keratomileusis for all levels of myopia. Br J Ophthalmol. 2006;90:20-23.

11. Mohamed Dirani TC, Joanne Yau, et al. Long-term refractive outcomes and stability after excimer laser surgery for myopia. J Cataract Refract Surg. 2010;36:1709-1717.

12. Sekundo W. BK, Mattausch P., et al. Six-year follow-up of laser in situ keratomileusis for moderate and extreme myopia using a first-generation excimer laser and microkeratome. J Cataract Refract Surg. 2003;29:1152-1158.

13. Hassan Hashemi MK, Mohammad Miraftab, Kazem Mohammad, Akbar Fotouhi. The Association Between Refractive Errors and Cataract: The Tehran Eye Study. Middle East Afr J Ophthalmol. 2011;18(2):154-158.

14. Arturo S. Chayet KKA, Miguel Montes, et al. Regression and Its Mechanisms after Laser In Situ Keratomileusis in Moderate and High Myopia. Ophthalmology. 1998;105(7):1194-1199.

15. Zalentein WN TT, Holopainen. Seven-year follow-up of LASIK for myopia. J Cataract Refract Surg. 2009;25(3):312-318.

16. Gerard Sutton ML, Christopher Hodge. Laser in situ keratomileusis in 2012: a review. Clinical and Experimental Optometry. 2014;97:18-29. 
17. Daphne CY Han JC, Hla Myint Htoon, et al. Comparison of outcomes of conventional WaveLight Allegretto Wave and Technolas excimer lasers in myopic laser in situ keratomileusis. Clinical Ophthalmology 2012;6:1159-1168.

18. Melissa D. Bailey KZ. Outcomes of LASIK for Myopia with FDA-Approved Lasers. Cornea. 2007;26(3):246-254. 